\title{
Adaptación transcultural del instrumento Orthognathic Quality of Life questionnaire al español para el estudio de la calidad de vida de pacientes con anomalías dentofaciales. Fase I
}

\section{Cross-cultural adaptation of the Spanish language version of the Orthognathic Quality of Life questionnaire for the assesment of quality of life in dentofacial deformities. Phase I.}

\author{
C. Caro Vasquez*, J. Castellanos Toro**, R. Pedraza Alarcón***, F. Sierra Matamoros****, \\ P. Ramírez Zea*****, M. Pinzón Navarro******, É. León Guzmán****
}

\section{RESUMEN}

Antecedentes. La cirugía ortognática es una opción terapéutica que busca parámetros de simetría, función masticatoria, respiratoria, e indirectamente mejorar el estado emocional de pacientes con anomalías dentofaciales. El instrumento Orthognathic Quality Of Life Questionnaire (OQLQ) permite realizar una evaluación subjetiva amplia y del nivel de calidad de vida pre y postquirúrgica, por lo que es necesario realizar el proceso de traducción al español y la adaptación cultural al contexto colombiano.

Métodos. Posterior a la autorización del autor original se realizaron traducciones directas, obtención de una versión preliminar, traducciones inversas y una prueba piloto con 15 pacientes colombianos, mayores de 16 años, con anomalía dentofacial que asistieron al hospital de San José. Se aplicó la metodología del grupo de calidad de la European Organization for Research and Treatment of Cancer (EORTC) para garantizar que la versión en español de la escala sea equivalente conceptualmente a la original.

Resultados. Las traducciones directas fueron consistentes, sin embargo, se hicieron ajustes para obtener la versión preliminar; las traducciones inversas fueron similares a la versión original de la escala. La prueba piloto, realizada en una muestra heterogénea de 15 pacientes, llevó al ajuste de la sintaxis de un ítem. De esta manera se obtuvo la versión en español del cuestionario que en fase posterior se validará.

Conclusiones. La realización de este estudio permitió la adaptación de un cuestionario entendible en el contexto colombiano, garantizando una equivalencia lingüística y conceptual entre esta versión y la versión original.

* $\quad$ Fellow cirugía maxilofacial, Fundación Universitaria de Ciencias de la Salud - Hospital de San José de Bogotá.

** Residente de tercer año de otorrinolaringología, Fundación Universitaria de Ciencias de la Salud Hospital de San José de Bogotá, Colombia.

*** Profesor Titular. Departamento de Otorrinolaringología y cirugía Maxilofacial, Fundación Universitaria de Ciencias de la Salud - Hospital de San José de Bogotá, Colombia.

**** Instructor asistente. División de investigaciones. Fundación Universitaria de Ciencias de la Salud- Hospital de San José de Bogotá, Colombia.

***** Residente de primer año de otorrinolaringología, Fundación Universitaria de Ciencias de la Salud - Hospital de San José de Bogotá.

******Profesor asociado Departamento de Otorrinolaringología y cirugía Maxilofacial, Fundación Universitaria de Ciencias de la Salud - Hospital de San José. Bogotá. 
PALABRAS CLAVE: Cirugía ortognática, maloclusión, calidad de vida.

\begin{abstract}
Introduction: Orthognathic surgery is a therapeutic option that seeks parameters of symmetry, masticatory function, breathing, and improves the emotional state of patients with dentofacial anomalies. The questionnaire Orthognathic Quality of Life Questionnaire (OQLQ) allows to make a subjective evaluation and the level of quality of life before and after surgery, so it is necessary to perform the translation into Spanish and the cultural adaptation to the Colombian context.

Methods: After the authorization of the original author, direct translations were made obtaining a preliminary version, reverse translations and a pilot test was accomplished with 15 Colombian patients over 16 years of age with a dentofacial anomaly who attended the San José hospital in Bogotá. The methodology of the quality group of the European Organization for Research and Treatment of Cancer (EORTC) was applied to ensure that the Spanish version of the scale is conceptually equivalent to the original.

Results: The direct translations were consistent, however, adjustments were made to obtain the preliminary version; the inverse translations were similar to the original version of the scale. The pilot test, carried out on a heterogeneous sample of 15 patients led to the adjustment of the syntax of one item. In this way, the Spanish version of the questionnaire was obtained.

Conclusion: The realization of this study allowed the adaptation of an understandable questionnaire in the Colombian context, guaranteeing a linguistic and conceptual equivalence between this version and the original version.
\end{abstract}

KEY WORDS: Orthognathic surgery, malocclusion, quality of life.

Fecha de recepción: 28 de febrero de 2019

Fecha de aceptación: 20 de marzo de 2019

C. Caro Vasquez, J. Castellanos Toro, R. Pedraza Alarcón, F. Sierra Matamoros, P. Ramírez Zea, M. Pinzón Navarro, É. León Guzmán. Adaptación transcultural del instrumento Orthognathic Quality of Life questionnaire al español para el estudio de la calidad de vida de pacientes con anomalías dentofaciales. Fase I. 2019; 35, (3): 119-129.

\section{INTRODUCCIÓN}

Las anomalías dentofaciales son malformaciones del maxilar o la mandíbula, con alteración en su forma, tamaño o posición, generando alteraciones físicas y funcionales de la masticación, habla, respiración y desempeño social ${ }^{(1,2)}$. Se reporta que afectan al $20 \%$ de la población en general y se asocia a morbilidades como apnea del sueño y disfunción de la articulación temporomandibular (ATM) ${ }^{(3,4)}$.

El tratamiento convencional incluye tratamiento de ortodoncia, cirugía ortognática o ambos. La ortodoncia pre quirúrgica, cirugía ortognática y ortodoncia posoperatoria generalmente produce resultados satisfactorios tales como descompensación dental con apropiada coordinación del arco dental y permite en caso de cirugía predicción segura de los movimientos maxilares o mandibulares ${ }^{(5)}$.

El enfoque tradicional de los profesionales sani- tarios en la práctica clínica generalmente no incluye aspectos sociales, físicos, personales o de percepción de la salud del paciente. La valoración de la calidad de vida en los procedimientos sanitarios ofrece al profesional de la salud nueva información sobre el actuar de su práctica médica. La disparidad entre los resultados clínicos y la puntuación de calidad de vida puede sugerir la necesidad de terapias alternativas o la existencia de más de una alteración, lo cual conlleva a contar con instrumentos de medida válidos y confiables para esta evaluación ${ }^{(6)}$.

En el Hospital de San José, en Bogotá y en general en Colombia, no se cuenta con una herramienta que permita realizar una valoración subjetiva de la calidad de vida de los individuos con este tipo de alteración, a pesar de estar reportado en la literatura instrumentos para medir dichas características.

El cuestionario OQLQ fue desarrollado y validado 
por Cunningham en el Reino Unido, en los años 2000 y 2002 respectivamente ${ }^{(7)}$. Sus 22 ítems valoran diferentes aspectos de las deformidades dentofaciales y el impacto que su tratamiento puede tener en la vida del paciente, divididas en 4 dominios (aspectos sociales, estética facial, funciones orales y conciencia de la deformidad facial) ${ }^{(8)}$.

El cuestionario de calidad de vida ortognático usa una calificación de escala de 4 puntos y está codificado de la siguiente manera: de 1 o "le molesta un poco" a 4 o "le molesta mucho", siendo 2 y 3 en el medio; y NA o "la declaración no se aplica a usted o no le molesta para nada". La puntuación del OQLQ se realiza mediante la adición de elementos individuales dentro de los dominios. Un puntaje OQLQ total puede variar de 0 a 88 . Una puntuación más alta indica una calidad de vida más pobre y una puntuación inferior indica mejor calidad de vida posoperatoria ${ }^{(9,10)}$.

Se ha empleado para comparar sus valores pre y postoperatorios y determinar si hay mejoría tras la finalización del tratamiento o, por el contrario, el paciente percibe insatisfacción por el mismo. Este cuestionario ha sido adaptado a otras culturas y se utiliza en varios países, actualmente se encuentra validado en los idiomas inglés, alemán, japonés, portugués y serbio ${ }^{(2,11,12)}$. Duarte et al, en Chile inició el proceso de adaptación transcultural para lograr la equivalencia de significados entre la cultura en la que se desarrolló el cuestionario y la versión en español chileno, pero aún no ha sido validada ${ }^{(13)}$.

El proceso de adaptación transcultural va más allá de la simple realización de la traducción de un instrumento, este procedimiento incluye el poder de asegurar la equivalencia tanto conceptual como lingüística de la versión que es traducida con la original ${ }^{(5)}$. Pese a que existen algunas variaciones en el proceso, se reconocen los lineamientos generales como los desarrollados por la European Organization for Research and Treatment of Cancer (EORTC) ${ }^{(14)}$ y la propuesta de Beaton ${ }^{(15)}$, los cuales se basan en elaborar traducciones directas de la lengua original a la lengua destino, después traducciones inversas para asegurar la equivalencia semántica y, posteriormente en la realización de pruebas piloto con los sujetos destinatarios del instrumento para revisar el parafraseo y posibles dificultades con el entendimiento de los ítems ${ }^{(16)}$.

El propósito de esta investigación fue realizar la traducción y adaptación cultural del cuestionario de calidad de vida al contexto colombiano, siguiendo lineamientos metodológicos que garanticen la equivalencia de conceptos, para en una fase posterior iniciar la validación de la misma.

\section{MATERIAL Y MÉTODOS}

Se realizó un estudio de adaptación transcultural del cuestionario del inglés al español colombiano. Este estudio se basó en la metodología propuesta por el grupo de calidad de vida de la European Organization for Research and Treatment of Cancer $(\text { EORTC })^{(14)}$ para garantizar que la versión en español del cuestionario tenga equivalencia conceptual con la original, que sea comprensible a personas con distintos niveles de escolaridad, aceptable culturalmente e inofensiva; por lo tanto, se constituyó un grupo de trabajo para la adaptación constituido por un médico especialista en otorrinolaringología, un médico especialista en cirugía maxilofacial, un odontólogo y un psicólogo con formación en epidemiología clínica y se siguieron las siguientes fases, representadas en la figura 1:

Solicitud de autorización: Se contactó a la autora original del cuestionario, Susan Cunningham, vía

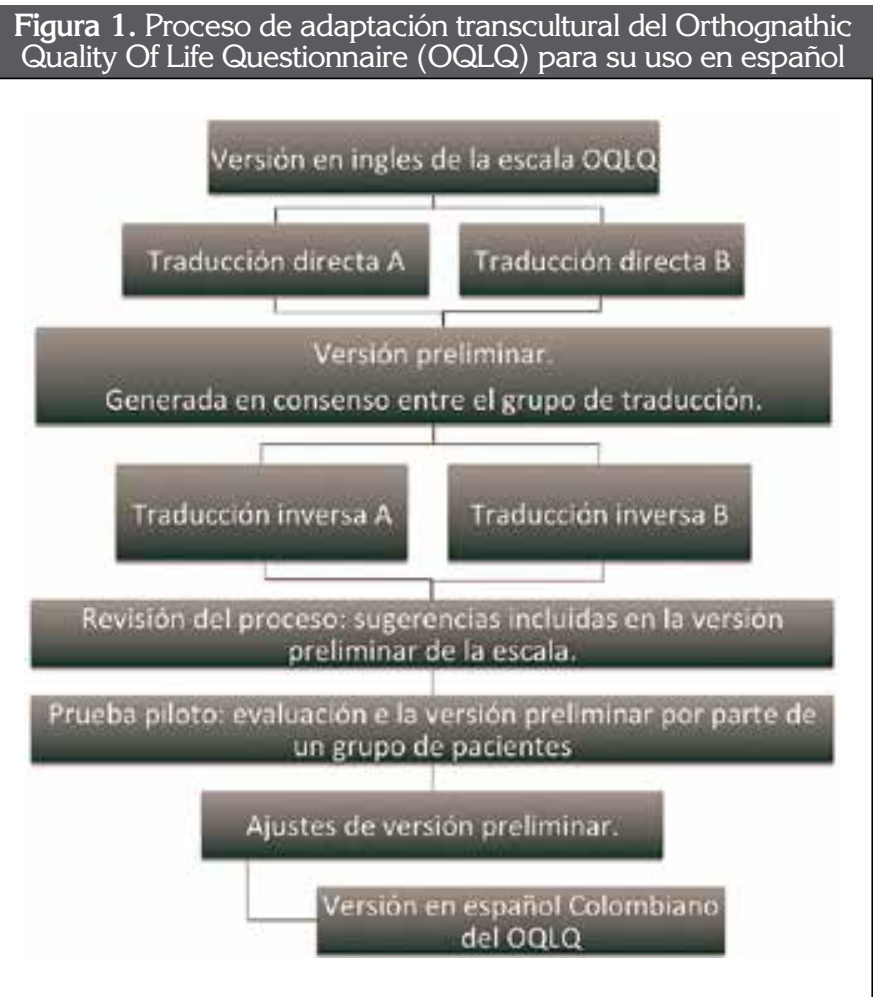


correo electrónico quien brindó su autorización por escrito el uso de la escala para su adaptación transcultural y validación.

Traducción directa: Dos colombianos (uno del área de la salud) con buena fluidez del idioma inglés realizaron dos traducciones directas del inglés al español colombiano de las instrucciones, los ítems y sus posibles opciones de respuesta. Ambos procesos de traducción fueron realizados de manera independiente.

Versión preliminar: El grupo de trabajo para la adaptación transcultural comparó las dos traducciones directas con el objetivo de analizar y resolver discrepancias entre las mismas y posteriormente generar una versión preliminar del cuestionario. La discusión se basó en los siguientes criterios: si las traducciones eran idénticas, no se hizo ninguna modificación; si existió alguna disimilitud, se escogió la traducción cuya significación tuviera mayor semejanza con la versión original; se seleccionó la traducción que cumpliera las siguientes apreciaciones ${ }^{(17)}$ :

- Reflejó la definición conceptual y el significado de la escala original.

- Reflejó el énfasis del texto original.

- Fue fácil de entender para personas sin conocimiento médico.

- Fue fácil de entender para personas de distintos niveles educativos.

- Fue lo más cercana al texto original, no añadió ni cambió información.

- Se leyó de la manera más natural.

- Fue culturalmente apropiada.

- Su sintaxis fue correcta.

- Incluyó todas las palabras clave.

- El vocabulario fue consistente a través de toda la traducción.

Una vez escogida la mejor versión para cada ítem se hicieron ajustes cuando fue necesario.

Traducción inversa: Las traducciones inversas del español colombiano al inglés fueron hechas por dos angloparlantes (nativos estadounidenses) con buena fluidez del español colombiano, a partir de la versión preliminar. Ambas traducciones fueron realizadas de manera independiente sin conocer la versión original de la escala. Estas versiones posteriormente fueron comparadas con la escala original por el grupo de trabajo para la adaptación.

Prueba piloto: Una vez definida y evaluada la versión preliminar, fue presentada de manera individual a 15 pacientes colombianos, hablantes nativos del español, mayores de 16 años, diagnosticados con anomalía dentofacial en el hospital de San José, candidatos a tratamiento quirúrgico con cirugía ortognática o ya quienes ya habían sido intervenidos. Los pacientes dieron su asentimiento verbal para participar en el estudio, posteriormente leyeron el cuestionario y diligenciaron un formato en el cual evaluaban si cada uno de los ítems y las instrucciones del cuestionario eran incomprensibles, confusos, ofensivos o molestos, tenían vocablos difíciles, o si era posible una redacción con un parafraseo mejor. En aquellos ítems en los que el 10\% o más de los pacientes indicaron alguno de los aspectos mencionados, se revisó la redacción, la propuesta de parafraseo y los comentarios de los pacientes y se realizaron los ajustes que aplicaron sin cambiar el sentido original de la escala.

Con la finalización de la prueba piloto se obtuvo la versión en español colombiano del cuestionario OQLQ. La presente investigación fue aprobada por el comité de investigaciones del hospital San José.

\section{RESULTADOS}

\section{TRADUCCIÓN DIRECTA}

Las traducciones directas se presentan en la tabla 1. Estas fueron conceptualmente iguales, sin embargo, se seleccionó la traducción A por tener un vocabulario y una sintaxis más apropiada que permite ser leída de manera natural por el examinado, con ligeras modificaciones obtenidas de la traducción $B$.

Las traducciones directas de los ítems 2, 3, 19 y 20 tuvieron una redacción idéntica, esto quiere decir que se emplearon los mismos vocablos y parafraseo semejante.

Las traducciones directas de la introducción y los 


\section{ESCALA ORIGINAL EN INGLÉS}

Please read the following statements carefully. In order to find out how important each of statements is to you, please circle 1. 2.3.4. or N/A where:

1 Means it bother you a Little

4 Means it bother you a lot

2+3 Lie between these statements

N/A Means the statements does not apply to yoyo $r$ does not bother you at all

1. I am self-conscious about the appearance of my teeth.

2. I have problems biting.

3. I have problems chewing.

\section{TRADUCCIÓN A}

Por favor lea cuidadosamente los siguientes enunciados. Con el fin de averiguar qué tan importante es para usted cada una de las siguientes afirmaciones, por favor marque 1,2,3,4 o N/A según aplique en donde: 1. Significa "le molesta poco"

4. Significa "le molesta mucho"

2+3. Se encuentra entre estas afirmaciones

N/A. Significa las afirmaciones no aplican

para usted o no le molestan en absoluto

Soy cuidadoso(a) acerca de la apariencia de mis dientes.

tengo problemas al morder

Tengo problemas para masticar

\section{TRADUCCIÓN B}

Por favor lea las siguientes afirmaciones cuidadosamente. Para determinar que tan importante es cada afirmación es para usted, por favor circule 1 , 2, 3, 4 o N/A donde:

1 Significa que le molesta un poco

4 Significa q le molesta bastante

2+3. Se haya entre esas afirmaciones

N/A Significa que la afirmacion no aplica o no le

molesta para nada

Soy consciente de mí mismo acerca de la aparien-

cia de mis dientes

Tengo problemas al morder

\section{VERSIÓN PRELIMINAR}

Por favor lea cuidadosamente los siguientes enunciados. Para determinar qué tan importante es para usted cada una de las siguientes afirmaciones, por favor marque 1,2,3,4 o N/A, donde:

1 Significa "le molesta poco".

4 Significa "le molesta mucho".

$2+3$ Se encuentra entre estas afirmaciones.

N/A Significa que las afirmaciones no aplican para

usted o no le molestan para nada.

Soy consciente de la apariencia de mis dientes.

Tengo problemas al morder.

Tengo problemas para masticar.
4. There are some foods i avoid eating because the way my teeth meet makes it difficult.

5. I don't like eating in public places.

6. I get pains in my face or jaw.

7. I don't like seeing a side view of my face (profile).

8. I spend a lot of time studying my face in the mirror.

9. I spend a lot of time studying my teeth.

10. I dislike having my photograph taken.

11. I dislike being seen on video.

12. I often stare at other people's teeth.

13. I often stare at other people's faces.

14. I am self-conscious about my facial

Hay algunos alimentos que evito comer porque la forma en que mis dientes se juntan hace que sea difícil.

No me gusta comer en lugares públicos

Me duele la cara o la mandíbula

No me gusta ver una vista lateral de mi cara. (Perfil) Paso mucho tiempo revisando mi cara en el espejo. Paso mucho tiempo revisando mis dientes en el espejo.

No me gusta que me tomen fotos.

No me gusta verme en videos.

A menudo miro fijamente los dientes de las otras personas.

A menudo miro fijamente las caras de las appearance.

15. I try to cover my mouth when I meet people for the first time.

16. I worry about meeting people for the first time.

17. I worry that people will make hurtful com-

ments about my appearance.

18. I lack confidence when I am out socially.

19. I do not like smiling when I meet people.

20. I sometimes get depressed about my

appearance.

21. I sometimes think that people are staring at me.

22. Comments about my appearance really upset me, even when I know people are only joking.

otras personas.

Soy cuidadoso(a) de mi apariencia facial

Trato de cubrirme la boca cuando conozco gente

Me preocupa conocer gente nueva.

Me preocupa que la gente haga comentarios

hirientes sobre mi apariencia.

Me falta confianza cuando estoy fuera en ambientes sociales.

No me gusta sonreír cuando conozco gente.

A veces me deprime mi apariencia.

A veces creo que la gente se fija en mí.

Los comentarios sobre mi apariencia realmente me molestan, incluso cuando sé que la gente solo está bromeando
Tengo problemas para masticar

Hay comidas que evito por la dificultad q causa la forma en que mis dientes se encuentran

No me gusta comer en sitios públicos

Tengo dolor en la cara o la mandíbula

No me gusta ver mi cara de lado (perfil) 1

Paso mucho tiempo estudiando mi cara en el espejo.

Paso mucho tiempo estudiando mis dientes en el espejo.

No me gusta ser fotografiado

No me gusta verme en video

A menudo miro los dientes de los demás

A menudo miro fijamente los rostros de los demás

Hay alimentos que evito comer porque la forma en que mis dientes se juntan me lo dificulta.

No me gusta comer en lugares públicos.

Me duele la cara o la mandíbula.

No me gusta ver mi cara de lado (perfil).

Paso mucho tiempo analizando mi cara en el espejo.

Paso mucho tiempo analizando mis dientes.

Me disgusta que me tomen fotos.

Me disgusta verme en video.

A menudo me quedo mirando los dientes de otras personas.

A menudo me quedo mirando las caras de otras personas.
Soy consciente de mí mismo acerca de mi apariencia facial

Trato de cubrir mi boca cuando conozco gente por primera vez

Me preocupo al conocer gente por primera vez

Me preocupa que la gente haga comentarios dolorosos acerca de mi apariencia

Me falta autoconfianza cuando salgo socialmente

No me gusta sonreír cuando conozco gente

A veces me deprime mi apariencia

A veces pienso que la gente me mira fijamente

Los comentarios sobre mi apariencia me molestan, aun cuando sé que son en broma.

Soy consciente de la apariencia de mi cara. Trato de cubrirme la boca cuando conozco gente nueva.

Me preocupa conocer gente nueva.

Me preocupa que la gente haga comentarios hirientes sobre mi apariencia.

Me falta confianza cuando salgo a socializar.

No me gusta sonreír cuando conozco gente.

A veces me deprime mi apariencia.

A veces pienso que la gente se queda mirándome. Los comentarios sobre mi apariencia realmente me molestan, incluso cuando sé que las personas solo están bromeando. ítems 4, 5, 6, 7, 12, 13, 15, 16, 17, 18, 21 у 22 usaron las mismas palabras, y aunque mostraron modificaciones en la redacción, fueron apropiadas al contexto colombiano por lo que se escogió la que se consideraba con mayor posibilidad de ser comprendida por los pacientes.
Se identificaron vocablos distintos en las traducciones de los ítems 1, 8, 9, y se cambiaron palabras o frases en los ítems 10, 11 y 14 así:

- Ítem 1. La traducción A fue "soy cuidadoso(a) acerca de la apariencia de mis dientes" 
ESCALA ORIGINAL EN INGLÉS

1. I am self-conscious about the appearance of my teeth.

2. I have problems biting.

3. I have problems chewing.

4. There are some foods i avoid eating because the way my teeth meet makes it difficult.

5. I don't like eating in public places.

6. I get pains in my face or jaw.

7. I don't like seeing a side view of my face (profile)

\section{TRADUCCIÓN INVERSA A}

I'm self-conscious about my teeth.

I have difficulties biting.

I have difficulties chewing.

There are certain foods I avoid eating due to state of my teeth.

I dislike eating in public.

My face and/or jaw hurts.

I dislike seeing my face in profile.

I often spend time analyzing my face in the mirror.

\section{TRADUCCIÓN INVERSA B}

I am aware of the appearance of my teeth.

I have issues when I bite.

I have issue when I chew.

I avoid certain foods because of the way my teeth are aligned make it hard for me to eat.

I dont like to eat in public places.

My face or jaw hurts.

I do not like my profile.

I spend a lot of time analyzing my face in the
8. I spend a lot of time studying my face in the mirror.

9. I spend a lot of time studying my teeth.

10. I dislike having my photograph taken.

11. I dislike being seen on video.

12. I often stare at other people's teeth.

13. I often stare at other people's faces.

14. I am self-conscious about my facial appearance.

15. I try to cover my mouth when I meet people for the first time.

I often spend time analyzing my teeth. I hate getting pictures taken.

I hate seeing myself on video

I often find myself looking at the teeth of others.

I often find myself looking at the faces of others.

I'm very aware of my facial appearance.

When meeting new people, I cover my mouth.

I dread meeting new people.

\section{mirror.}

I spend a lot of time analyzing my teeth.

I dont like when people take pictures of me.

I dislike seeing myself on video.

I often look at other peoples teeth.

I often find myself looking at other peoples

faces.

I am aware of the appearance of my face

I tend to cover my mouth when i meet new people.
16. I worry about meeting people for the first time. 17. I worry that people will make hurtful comments about my appearance.

18. I lack confidence when I am out socially.

19. I do not like smiling when I meet people.

20. I sometimes get depressed about my appearance.

21. I sometimes think that people are staring at me.

22. Comments about my appearance really upset me, even when I know people are only joking.

I worry that people say hurtful things about my appearance.

I lack confidence in social interactions.

I avoid smiling when I know people.

My appearance is a source of my unhappiness.

I often worry that people stare at me.

I am hurt by what people say about my

appearance, even when I know they are joking.

I worry about meeting new people.

I worry that people make hurtful comments about my appearance.

I lack confidence when I socialize.

I dont like to smile when I meet new people.

At times, i get depressed about my appea-

rance.

Sometimes i think people stare at me.

Comments about my appearance really bother me, even when I know people are joking. y la B, "soy consciente de mí mismo acerca de la apariencia de mis dientes". No se eligió la traducción A dado que la palabra "cuidadoso" conceptualmente indica "que se protege o conserva una cosa con esmero o interés" ${ }^{(18)}$,por lo que se realizó un ligero cambio de la traducción $\mathrm{B}$, anulando la frase "de mi mismo acerca" por considerarse redundante; de esta manera la traducción preliminar de este ítem fue "soy consciente de la apariencia de mis dientes", la cual es gramaticalmente correcta y es más comprensible para los examinados.

- Ítem 8. Las dos traducciones emplearon el verbo revisar o estudiar ("paso mucho tiempo revisando mi cara en el espejo", "paso mucho tiempo estudiando mi cara en el espejo"); en la versión preliminar se usó "analizar o detallar" ya que conceptualmente refleja mejor la acción que haría un paciente con anomalía dentoesqueletica que comprometa su estado físico o estético.

- Ítem 9. Las dos traducciones emplearon los verbos "revisar" o "estudiar" los cuales no refle- jan de manera más cercana la acción del paciente con anomalía estética dental, quien analiza o detalla el estado de sus dientes; por lo tanto en la versión preliminar se empleó el verbo "analizar o detallar" y se omitió "en el espejo" que no está en la versión original del instrumento.

- Ítem 10. Se escogió la traducción A ("no me gusta que me tomen fotos") dado que culturalmente es más adecuado el término "tomar fotos" que "fotografiar" (empleado en la traducción B) y se cambió "no me gusta" a "me disgusta" porque es más fiel a la redacción original (I don't like versus I dislike) y porque así el ítem conservó su redacción afirmativa.

-Ítem 11. Aunque las traducciones fueron idénticas, se cambió la expresión "no me gusta" a "me disgusta" ya que es más fiel a la redacción original (I don't like versus I dislike) y porque así el ítem conservó su redacción afirmativa.

- Ítem 14. Se tomaron partes de ambas traducciones, y se cambió "apariencia facial" por 


\begin{tabular}{|c|c|c|c|c|c|c|c|c|c|}
\hline ID & $\begin{array}{l}\text { Edad } \\
\text { (años) }\end{array}$ & Género & Ocupación & $\begin{array}{l}\text { Motivo de } \\
\text { consulta }\end{array}$ & $\begin{array}{c}\text { ANGLE } \\
\text { pre quirúrgico }\end{array}$ & $\begin{array}{c}\text { Anomalía } \\
\text { oclusal }\end{array}$ & $\begin{array}{l}\text { Hiperplasia } \\
\text { condilar }\end{array}$ & Cirigía & $\begin{array}{c}\text { Tipo de } \\
\text { cirugía }\end{array}$ \\
\hline 1 & 20 & Femenino & Estudiante & Físico & III & Cruzada & $\mathrm{Si}$ & Si & Bimaxilar \\
\hline 2 & 19 & Masculino & Estudiante & Masticación & III & Abierta & No & No & NA \\
\hline 3 & 22 & Femenino & Estudiante & Físico & I & Cruzada, abierta & $\mathrm{Si}$ & $\mathrm{Si}$ & Bimaxilar \\
\hline 4 & 33 & Femenino & Profesional & Físico & III & Cruzada & Si & $\mathrm{Si}$ & Bimaxilar \\
\hline 5 & 27 & Masculino & Profesional & Dolor ATM & II & Sin dato & No & No & NA \\
\hline 6 & 20 & Masculino & Profesional & Físico & III & Cruzada, abierta & $\mathrm{Si}$ & No & NA \\
\hline 7 & 26 & Masculino & Profesional & Físico & III & Cruzada & No & No & NA \\
\hline 8 & 31 & Femenino & Otro & Físico & III & Cruzada & Si & No & NA \\
\hline 9 & 19 & Femenino & Estudiante & Dolor ATM & I & Cruzada & Si & No & Bimaxilar \\
\hline 10 & 35 & Femenino & Otro & Físico & II & Cruzada, Borde a borde & Si & No & NA \\
\hline 11 & 27 & Masculino & Otro & Dolor ATM & III & Cruzada & No & $\mathrm{Si}$ & Bimaxilar \\
\hline 12 & 17 & Femenino & Estudiante & Dolor ATM & III & Cruzada & $\mathrm{Si}$ & No & NA \\
\hline 13 & 21 & Masculino & Estudiante & Físico & III & Cruzada & No & $\mathrm{Si}$ & Bimaxilar \\
\hline 14 & 21 & Masculino & Estudiante & Masticación & III & Abierta & No & $\mathrm{Si}$ & Bimaxilar \\
\hline 15 & 25 & Masculino & Otro & Dolor ATM & III & Abierta & No & No & NA \\
\hline
\end{tabular}

ATM: Articulación Temporomandibular, NA: No Aplica

"la apariencia de mi cara", un término culturalmente más utilizado en nuestro idioma, quedando la versión preliminar redactada así: "soy consciente de la apariencia de mi cara".

Con las modificaciones mencionadas anteriormente se redactó la versión preliminar de la escala en español, la cual puede observarse en la tabla 1.

\section{TRADUCCIÓN INVERSA}

Las dos traducciones fueron gramaticalmente similares entre sí y usaron sinónimos en algunas palabras. Las dos redacciones no hicieron cambios en el objetivo de la pregunta y, aunque se usaron algunos cambios de conjugación y presente continuo, las nuevas traducciones conservaron su finalidad y fueron similares a la versión original del instrumento (ver tabla 2); por lo tanto no se requirió algún ajuste a la versión preliminar.

\section{PRUEBa PILOTO}

La prueba piloto fue realizada en abril de 2018 con 15 pacientes, correspondientes a 7 mujeres (46.6\%) y 8 hombres (53.3\%) con una edad media de 24 años (DE: 5.5 años). En la tabla 3 se muestran los datos sociodemográficos de los pacientes, que en su mayoría fueron estudiantes (53.3\%), consultaron más frecuentemente por su aspecto físico (57.1\%), tenían una clasificación de Angle prequirúrgica en su mayoría de clase III (73.3\%) y una anomalía oclusal tipo mordida cruzada en el $57.1 \%$, la hiperplasia condilar se presentó en 8 pacientes (57.1\%) y de la totalidad de la muestra ya habían sido operados el $46 \%$ (7 pacientes). El tiempo empleado en la aplicación del instrumento fue de 30 minutos.

\begin{tabular}{lllll} 
Item & \multicolumn{3}{l}{ Tabla 4. Resultados de la prueba piloto } \\
& Dificultad & Confusión & $\begin{array}{l}\text { Palabras } \\
\text { difíciles }\end{array}$ & $\begin{array}{l}\text { Palabras } \\
\text { ofensivas }\end{array}$ \\
\hline 1 & 0 & 0 & 0 & $1(6.6 \%)$ \\
2 & 0 & 0 & 0 & 0 \\
3 & 0 & $2(13.3 \%)^{1}$ & 0 & 0 \\
4 & 0 & $6(40 \%)^{2}$ & 0 & 0 \\
5 & 0 & 0 & 0 & 0 \\
6 & 0 & 0 & $1(6.6 \%)$ & 0 \\
7 & 0 & 0 & 0 & 0 \\
8 & 0 & 0 & 0 & 0 \\
9 & 0 & 0 & 0 & 0 \\
10 & 0 & 0 & 0 & 0 \\
11 & 0 & 0 & 0 & 0 \\
12 & 0 & 0 & 0 & 0 \\
13 & 0 & 0 & 0 & 0 \\
14 & 0 & 0 & 0 & $7(46 \%)^{3}$ \\
15 & 0 & 0 & 0 & 0 \\
16 & 0 & 0 & 0 & 0 \\
17 & 0 & 0 & 0 & $1(6.6 \%)$ \\
18 & 0 & 0 & 0 & $1(6.6 \%)$ \\
19 & 0 & 0 & 0 & 0 \\
20 & 0 & 0 & 0 & $1(6.6 \%)$ \\
21 & 0 & 0 & 0 & $1(6.6 \%)$ \\
22 & 0 & 0 & 0 & $2(13.3 \%)$ \\
\hline 15 & 0 & 0 & 0 & \\
\hline
\end{tabular}

1 Se propuso cambiar la palabra masticar por ingerir; sin embargo, no se incluyó el cambio porque se refirió a un concepto diferente. ${ }^{2}$ Se sugirió y se incluyó el cambio de "juntar los dientes" a "mordida". ${ }^{3}$ Los pacientes consideraron que el parafraseo de la versión preliminar "soy consciente" puede relacionarse con un aspecto negativo de la cara; sin embargo, el cambio propuesto "me gusta la apariencia de mi cara" no reflejó el concepto original; como el primer ítem emplea las mismas palabras ("soy consciente") y no fue mal evaluado por la mayoría de los pacientes, se dejó la redacción propuesta en la versión original.

La prueba piloto (tabla 4) mostro que en los ítems $2,5,7,8,9,10,11,12,13,15,16$ y 19 no hubo ningún paciente que manifestara dificultad en comprender los ítems, no manifestaron confusión en la pregunta, no encontraron palabras difíciles ni les pareció ofensivo. En el ítem 3, dos personas men- 


\section{Tabla 5. Versión en español colombiano de la escala OQOL}

\section{Instrucciones}

Por favor lea cuidadosamente los siguientes enunciados. Para determinar qué tan importante es para usted cada enunciado, por favor marque 0,1,2,3, o 4 donde: 0 significa "nada" o "no aplica en usted" / 1 significa "un

\begin{tabular}{lccccc}
\hline & Nada & Un poco & Algo & Mucho & Muchísimo \\
\hline 1. Soy consciente de la apariencia de mis dientes. & 0 & 1 & 2 & 3 & 4 \\
2. Tengo problemas al morder & 0 & 1 & 2 & 3 & 4 \\
3. Tengo problemas para masticar & 0 & 1 & 2 & 3 & 4 \\
4. Hay alimentos que evito comer porque mi mordida me lo dificulta. & 0 & 1 & 2 & 3 & 4 \\
5. No me gusta comer en lugares públicos & 0 & 1 & 2 & 3 & 4 \\
6. Me duele la cara o la mandíbula & 0 & 1 & 2 & 3 & 4 \\
7. No me gusta ver mi cara de lado (perfil) & 0 & 1 & 2 & 3 & 4 \\
8. Paso mucho tiempo analizando mi cara en el espejo & 0 & 1 & 2 & 3 & 4 \\
9. Paso mucho tiempo analizando mis dientes & 0 & 1 & 2 & 3 & 4 \\
10. Me disgusta que me tomen fotos & 0 & 1 & 2 & 3 & 4 \\
11. Me disgusta verme en video & 0 & 1 & 2 & 3 & 4 \\
12. A menudo me quedo mirando los dientes de otras personas & 0 & 1 & 2 & 3 & 4 \\
13. A menudo me quedo mirando las caras de otras personas. & 0 & 1 & 2 & 3 & 4 \\
14. Soy consciente de la apariencia de mi cara. & 0 & 1 & 2 & 3 & 4 \\
15. Trato de cubrirme la boca cuando conozco gente nueva & 0 & 1 & 2 & 3 & 4 \\
16. Me preocupa conocer gente nueva & 0 & 1 & 2 & 3 & 4 \\
17. Me preocupa que la gente haga comentarios hirientes sobre mi apariencia & 0 & 1 & 2 & 3 & 4 \\
18. Me falta confianza cuando salgo a socializar & 0 & 1 & 2 & 3 & 4 \\
19. No me gusta sonreír cuando conozco gente & 0 & 1 & 2 & 3 & 4 \\
21. A veces pienso que la gente se queda mirándome & 0 & 1 & 2 & 3 & 4 \\
22. Los comentarios sobre mi apariencia realmente me molestan & & & & & \\
incluso cuando sé que las personas solo están bromeando & 0 & 1 & 2 & 3 & 4 \\
\hline
\end{tabular}
poco" / 2 significa "algo" / 3 significa "mucho" / 4 significa "muchísimo".

En relación con las instrucciones, los participantes manifestaron algunas dificultades. La primera se relaciona con las opciones intermedias, 2 o 3, en las que no hay una descripción clara, a diferencia de la 1 ("le molesta poco") y la 5 ("le molesta mucho") por lo que es más fácil escoger estos extremos. La segunda se relaciona con la aplicación de las opciones "le molesta poco" y "le molesta mucho" ante enunciados que inician con expresiones como "me preocupa conocer...", "me disgusta...", "me preocupa...", "no me gusta..." con los cuales cionaron sentir confusión entre las palabras morder y masticar por lo que fue llevado a consenso en donde se decidió que no representaba un factor de confusión en la mayoría de los encuestados por lo que no se hicieron cambios de la pregunta original. De los ítems 1, 17, 18, 20, 21 y 22 no se reportaron palabras ofensivas, pero si sensibilidad en el tema específicamente en un paciente por lo que no se hizo ninguna modificación en el ítem.

Finalmente, los ítems que presentaron controversia fueron el 4 y el 14 . En el ítem 4 , el $40 \%$ de los pacientes reportaron no entender adecuadamente "la forma en que mis dientes se juntan" y sugirieron la palabra "mordida" en vez de "se junta" por lo que se hizo consenso dejando finalmente "Hay alimentos que evito comer porque mi mordida me lo dificulta". En cuanto al ítem 14, siete pacientes refirieron ser una pregunta ofensiva ya que generaliza un aspecto negativo de toda la cara y sugirieron la expresión "me gusta"; se realizó consenso en el grupo de adaptación transcultural el cual concluyó que el cambio propuesto "me gusta la apariencia de mi cara" no reflejó el concepto original; como el primer ítem empleó las mismas palabras ("soy consciente") y no fue mal evaluado por la mayoría de los pacientes, se dejó la redacción propuesta en la versión preliminar. los pacientes ya se identifican y prefieren mencionar el grado en que los enunciados aplican en ellos. Por lo tanto, el grupo de adaptación transcultural revisó el objetivo de distintas opciones de respuestas en escalas tipo Likert, considerando apropiada una escala unipolar para medir el grado de constructo(19) y las opciones empleadas en la evaluación de la calidad de vida relacionada con la salud en otras patologías, como las de las escalas FACT-G y EORTC QLQ STO22 ya validadas en Colombia $(20,21)$ y propuso la redacción de las instrucciones propuestas en la escala FACT-G. Esta propuesta fue consultada con la autora de la escala quien estuvo de acuerdo con el cambio.

La versión definitiva con los cambios expuestos se presenta en la tabla 5. Se tomaron en cuenta los comentarios realizados por los pacientes, consenso entre los investigadores principales y la definición de las palabras usadas en la versión definitiva teniendo en cuenta el contexto colombiano.

\section{DISCUSIÓN}

La medición de la calidad de vida en salud es un tema emergente, el cual se ha ido consolidando con el desarrollo de metodologías estandarizadas para la creación de instrumentos que en su gran mayoría surgen 
en el idioma inglés. Se busca que un instrumento traducido sea capaz de lograr en la cultura objetivo el mismo efecto que tiene en el contexto original donde fue desarrollado. La falta de equivalencia intercultural obstaculiza la validez de la información recopilada, lo que imposibilita el uso adecuado del instrumento para estudiar el concepto planteado ${ }^{(22)}$.

Es frecuente encontrar diferentes versiones de un cuestionario traducido que el autor o autores originales no reconocen porque no se les contacta para pedir su consentimiento para la traducción y uso del cuestionario, por lo cual el presente estudio partió de la autorización de la Doctora Cunningham. Adicionalmente al adaptar los instrumentos se busca permitir la internacionalización de los datos, debido a que se pueden hacer comparaciones interculturales ya que la medición en diferentes sociedades lo permite, es así como la traducción y adaptación se convierte en un paso fundamental como el proceso original del desarrollo del instrumento ${ }^{(23)}$.

Al comparar la versión original del cuestionario de calidad de vida ortognático, con la versión consolidada de las traducciones inversas, el grupo de traducción pudo verificar que eran similares, por lo cual fue aplicado en la prueba piloto de pacientes. La realización de la prueba piloto fue realizada con individuos heterogéneos que representan la población que requiere este tipo de cirugías, vale la pena aclarar que es población joven (edad mínima de 17 años y un máximo de 33 años) como la reportada por la autora de la escala de mayores de 16 años o en la adaptación a portugués brasilero en que el promedio de edad de los pacientes fue de 25 años ${ }^{(23)}$.

Esta población joven se ve afectada en su apariencia física, por lo cual el motivo de consulta por esta causa fue del 57\%, sin embargo, la consulta por dolor articular e inconvenientes con la masticación también fueron reportados, al igual que la variabilidad en la clasificación de Angle y la participación de pacientes sin operar y operados.

Los ítems 4 y 14 fueron los más discutidos por los pacientes, por particularidades en la sintaxis, pero no en el entendimiento de las mismas, se hicieron los ajustes necesarios sugeridos. Los resultados en las adaptaciones de la población serbia no mostraron ítems que resultaran ofensivas o confusas, el $2 \%$ de los pacientes necesitaron ayuda para completar el cuestionario, aunque no especifican los ítems problema(24) . El 100\% de los pacientes manifestaron que el cuestionario fue entendible, no presentaron pérdida de datos ${ }^{(24)}$.

En el caso de la adaptación realizada al portugués brasilero existen dos publicaciones ${ }^{(23)}$, en una se presenta la metodología aceptada para la realización de adaptaciones transculturales, pero no hacen análisis de los resultados específicos del proceso. El segundo artículo reporta una metodología más detallada del proceso. El cuestionario en portugués demostró ser simple, con fácil entendimiento, preserva y es equivalente con su versión en inglés ${ }^{(11)}$.

Se encuentra como limitaciones de la adaptación la tendencia de los encuestados de responder la encuesta de forma personal y no a la evaluación objetiva de cada ítem. Para minimizar el efecto de esta limitación, antes de la realización de la encuesta se explicó el objetivo de las preguntas que fueron realizadas y se recordó durante el proceso.

Las motivaciones, percepciones y expectativas del paciente juegan un rol en el éxito de la cirugía y en los resultados psicológicos derivados de este ${ }^{(9)}$. La adaptación transcultural es el inicio para realizar la validación del cuestionario y así posteriormente poder medir la satisfacción del paciente justificando los costos biológicos y financieros que derivan de la atención en la práctica pública o privada.

La realización de este estudio permitió la adaptación de un cuestionario entendible en el contexto colombiano, garantizando una equivalencia lingüística y conceptual entre esta versión y la versión original. Los presentes hallazgos pueden ser útiles para los profesionales sanitarios de habla española, en las áreas de cirugía maxilofacial, otorrinolaringología, ortodoncia, entre otras.

Con los resultados de este estudio se puede iniciar la realización de las fases posteriores del proceso de validación de las propiedades psicométricas necesarias para evaluar la validez y confiabilidad del cuestionario para medir calidad de vida en pacientes con anomalía dentofacial hablantes del español.

\section{AGRADECIMIENTOS}

Agradecemos a Susan Cunningham autora del cuestionario, quien autorizó su uso para el estu- 
dio de adaptación transcultural y validación. A Dayanna Petrella y Juan Nicolás Kattan, por su colaboración en la realización de las traducciones directas. A Lilian Rodríguez y Héctor Amaya, quienes participaron con la elaboración de las traducciones inversas. Adicionalmente a los pacientes del Hospital San José, por su participación en la realización de la prueba piloto.

\section{DECLARACIÓN DE CONFLICTO DE INTERÉS}

No existe ningún conflicto de intereses por parte de los investigadores.

\section{DECLARACIÓN DE FINANCIACIÓN DEL PROYECTO}

La presente investigación no ha recibido ayudas específicas provenientes de agencias del sector público, sector comercial o entidades sin ánimo de lucro.

\section{BIBLIOGRAFIA}

1. Bedoya-Rodriguez A, Collo-Quevedo L, Gordillo-Melendez L, Yusti-Salazar A, Tamayo-Cardona J, Pérez-Jaramillo A, et al. Anomalías dentales en pacientes de ortodoncia de la ciudad de Cali, Colombia. (Dental anomalies in orthodontic patients in Cali, Colombia). CES Odontología. 2014;27(1):45-54.

2. Diaz-Reissner CV, Casas-García I, Roldán-Merino J. Calidad de Vida Relacionada con Salud Oral: Impacto de Diversas Situaciones Clínicas Odontológicas y Factores Socio-Demográficos. Revisión de la Literatura 2017;11(1):31-9.

3. Lam D, Laskin D. Oral And Maxillofacial Surgery Review, A study Guide. 1 ed. Chicago (US): Quintessence publisihing Co, Inc; 2015.

4. Fonseca $\mathrm{R}$, Walker $\mathrm{R}$, Barber $\mathrm{D}$, Powers $\mathrm{M}$, Frost D. Oral and Maxillofacial Trauma. 4 ed. Philadelphia: Elsevier Saunders; 2005.

5. Wang J, Chen W, Ni Z, Zheng M, Liang X, Zheng $Y$, et al. Timing of orthognathic surgery on the changes of oral health-related quality of life in Chinese orthognathic surgery patients. Am J Orthod Dentofacial Orthop. 2017;151(3):565-71.

6. Misrachi C, Espinoza I. Utilidad de las Mediciones de la Calidad de Vida Relacionada con la Salud. Revista Dental de Chile. 2005;96(2):28- 35.

7. Cunningham SJ, Garratt AM, Hunt NP. Development of a condition-specific quality of life measure for patients with dentofacial deformity: I. Reliability of the instrument. Community Dent Oral Epidemiol. 2000;28(3):195-201.

8. Lonic D, Pai B, Yamaguchi K, Chortrakarnkij P, Lin H-H, Lo L. Computer-Assisted Orthognathic Surgery for Patients with Cleft Lip/Palate: From Traditional Planning to Three-Dimensional Surgical Simulation. PLoS One. 2016;22(11(3)):e0152014.

9. Miguel JA, Palomares NB, Feu D. Life-quality of orthognathic surgery patients: the search for an integral diagnosis. Dental Press J Orthod. 2014;19(1):123-37.

10. Bortoluzzi MC, de Camargo Smolarek P, Claudino M, Campagnoli EB, Manfro R. Impact of Dentofacial Deformity on Quality of Life: Age and Gender Differences Evaluated Through OQLQ, OHIP and SF36. J Oral Maxillofac Res. 2015;6(3):e3. Epub 2015/06/30.

11. Bullinger M, Anderson R, Cella D, Aaronson N. Developing and evaluating cross-cultural instruments from minimum requirements to optimal models. Qual Life Res. 1993;2(6):451-9.

12. Bortoluzzi MC, Manfro R, Soares IC, Presta AA. Cross-cultural adaptation of the orthognathic quality of life questionnaire (OQLQ) in a Brazilian sample of patients with dentofacial deformities. Med Oral Patol Oral Cir Bucal. 2011;16(5):e694-9. Epub 2011/08/01.

13. Duarte $M V$, Villanueva $M$. Adaptación cultural del Orthognathic Quality Of Life (OQLQ): Informe Preliminar. XXXII Jornadas Chilenas de Salud Pública: Facultad de Medicina Universidad de Chile; 2017. 
14. Kuli冈 D, Bottomley A, Velikova G, Greimel E, Koller M, EORTC Quality of Life Group. ORTC Quality of life group translation procedure. 4 ed. Brussels (Belgium): EORTC; 2017.

15. Beaton DE, Bombardier C, Guillemin F, Ferraz MB. Guidelines for the process of cross-cultural adaptation of self-report measures. Spine (Phila Pa 1976). 2000;25(24):3186-91.

16. Sierra-Matamoros FA, Sánchez-Pedraza $R$, Ibañez-Antequera CI. Adaptación transcultural de la escala Daily Spiritual Experience Scale para su uso en Colombia. Revista colombiana de cancerología. 2013;17(4):149-57.

17. Koller M, Kantzer V, Mear I, Zarzar K, Martin M, Greimel E, et al. The process of reconciliation: evaluation of guidelines for translating quality-of-life questionnaires. Expert Rev Pharmacoecon Outcomes Res. 2012;12(2):189-97.

18. Real Academia Española. Diccionario de la lengua española / Real Academia Española. 23 ed. Barcelona, España: Espasa Libros; 2014. 2312 p.

19. Streiner DL, Norman GR, Cairney J. Health measurement scales : a practical guide to their development and use. Fifth edition. ed. Oxford: Oxford University Press; 2015. xiii, 399 pages $p$.

20. Ibáñez C, Sánchez R, Oliveros R. Validación para la utilización en Colombia de la escala EORTC QLQ-STO22 para la evaluación de la calidad de vida de pacientes con cáncer de estómago. Avances en Psicología Latinoamericana. 2015;33(3):397-411.

21. Sanchez R, Ballesteros M, Arnold BJ. Validation of the FACT-G scale for evaluating quality of life in cancer patients in Colombia. Qual Life Res. 2011;20(1):19-29.

22. Al-Asfour A, Waheedi M, Koshy S. Survey of patient experiences of orthognathic surgery: health-related quality of life and satisfaction. Int J Oral Maxillofac Surg. 2018. Epub 2018/01/17.

23. de Araújo AM, Miguel JA, Gava EC, de Oliveira $\mathrm{BH}$. Translation and cross-cultural adaptation of an instrument designed for the assessment of quality of life in orthognatic patients. Dental Press J Orthod. 2013;18(5):99-106.

24. Vucic L, Glisic B, Kisic-Tepavcevic D, Vucic U, Drulovic J, Pekmezovic T. Cross-cultural adaptation and validation of the disease specific questionnaire OQLQ in Serbian patients with malocclusions. Zdr Varst. 2016;55(3):166-73. Epub 2016/05/10.

\section{AUTOR DE CORRESPONDENCIA:}

Érika León Guzmán

Cl. 10 \#18-75, Bogotá, Colombia.

Dirección postal: 111411

División de investigaciones, Hospital de San José

Email: eleon1@fucsalud.edu.co

Teléfono: +573005961333 